\title{
Rare cause of recurrent fainting - multiple kinking and brachiocephalic origin of the left common carotid artery
}

\author{
Ewa Kurys-Denis ${ }^{1}$, Andrzej Prystupa ${ }^{2}$, Monika Wójtowicz ${ }^{2}$, Jerzy Mosiewicz² ${ }^{2}$ Witold Krupski ${ }^{1}$ \\ ${ }^{1}$ Department of Radiology, Medical University, Lublin, Poland \\ ${ }^{2}$ Department of Internal Medicine, Medical University, Lublin, Poland
}

Kurys-Denis E, Prystupa A, Wójtowicz M, Mosiewicz J, Krupski W. Rare cause of recurrent fainting - multiple kinking and brachiocephalic origin of the left common carotid artery. J Pre-Clin Clin Res. 2013; 7(1): 66-68.

\begin{abstract}
The case is presented of a 80-year-old male patient with a history of a few episodes of fainting. In the computed tomography of the head and CT angiography of the carotid arteries, an abnormal origin of the left common carotid artery from the brachiocephalic trunk and multiple, tortuous courses of cervical vessels were found.

The purpose of this article is to describe clinical advances in patients with cerebral insufficiency secondary to kinking of the carotid arteries, and to discuss the methods used in the diagnosis and treatment.
\end{abstract}

\section{Key words}

recurrent fainting, carotid kinking, carotid anomalies, common carotid artery

\section{INTRODUCTION}

Kinking of the artery is an infrequent clinical entity mostly described in the internal carotid artery. The embryological development of the aortic arch can produce numerous variations. Aortic arch anomalies are classified into five groups:

1) double aortic arch;

2) left aortic arch;

3) right aortic arch;

4) cervical aortic arch;

5) carotid anomalies $[1,2]$.

The ontogenetic explanation, clinical implications, and management options of these diseases are discussed in literature [3]. It is generally accepted that carotid anomalies can have important clinical implications, and arterial insufficiency as a result of kinking of the internal carotid artery can be a common finding with other signs of cerebral insufficiency $[1,4]$.

There are few English medical literature reports about the abnormal origin of the left common carotid artery and numerous kinkings as the cause of recurrent fainting. Having searched the medical literature, only two articles were found concerning common carotid artery coiling $[5,6]$ and one of common carotid artery tortuosity [7]. One of the articles reported a case of common carotid artery coiling, causing cerebrovascular insufficiency [6].

\section{CASE REPORT}

The 80 -year-old male patient was admitted to the Department of Internal Medicine because of a few episodes of fainting. The latest, short-lasting episode of fainting with a loss of consciousness was observed. The patient had a hypertensive

Address for correspondence: Ewa Kurys-Denis 2nd Department of Radiology, Medical University, Staszica 16, 20-081 Lublin, Poland

e-mail: ekurys@mp.pl

Received: 23 February 2013; accepted: 12 June 2013 disease which had been treated for many years. In the emergency room, blood pressure was $140 / 90 \mathrm{mmHg}$. Clinical examination, ECC, echocardiography, chest X-ray and blood test results were without any abnormality.

In the physical examination, there was no pulsatile mass in the neck, and the carotid pulsations on both sides were normal. Changes in the position of the head, however, induced neurological symptoms, such as dizziness and vertigo.

Computed tomography examination of the head with perfusion study, as well as CT angiography of the carotid arteries, were performed. The examinations showed an abnormal origin of the left common carotid artery from the brachiocephalic trunk, and many double and threefold kinking of the carotid and vertebral arteries.

Examination of the carotid arteries was performed using a spiral computed tomography angiography. Arterial enhancement was provided by the intravenous administration of $60 \mathrm{ml}$ of contrast material. After acquisition, images were post-processed at the workstations, producing VRT, MIP and MPR reconstructions. Image analysis showed an abnormal origin of the left common carotid artery from the short brachiocephalic trunk (about $18 \mathrm{~mm}$ long), located on the right side of the middle line of the body (Fig. 1). The brachiocephalic trunk did not show any significant changes. It is short to the site where the abnormal origin of the left common carotid artery was found. Beyond that, the brachiocephalic trunk was about $50 \mathrm{~mm}$ long. In addition to abnormal issue of the left carotid artery, there was an elongation of the artery itself. The artery coursed from the right to the left side of the middle line of the body, crossed the left subclavian artery, making a double circular configuration (two-stepped kinking), coursed further to the front, a little to the right, and up (second kinking) similar to the left subclavian artery (Fig. 2). The right common carotid artery coursed in a typical manner, but it made a big angular curve. In kinking, it produced a relative narrowing of the vessel (probably due to position of the body). Both the right and left subclavian arteries also had two-stepped kinking, The proximal part of the left vertebral artery was 


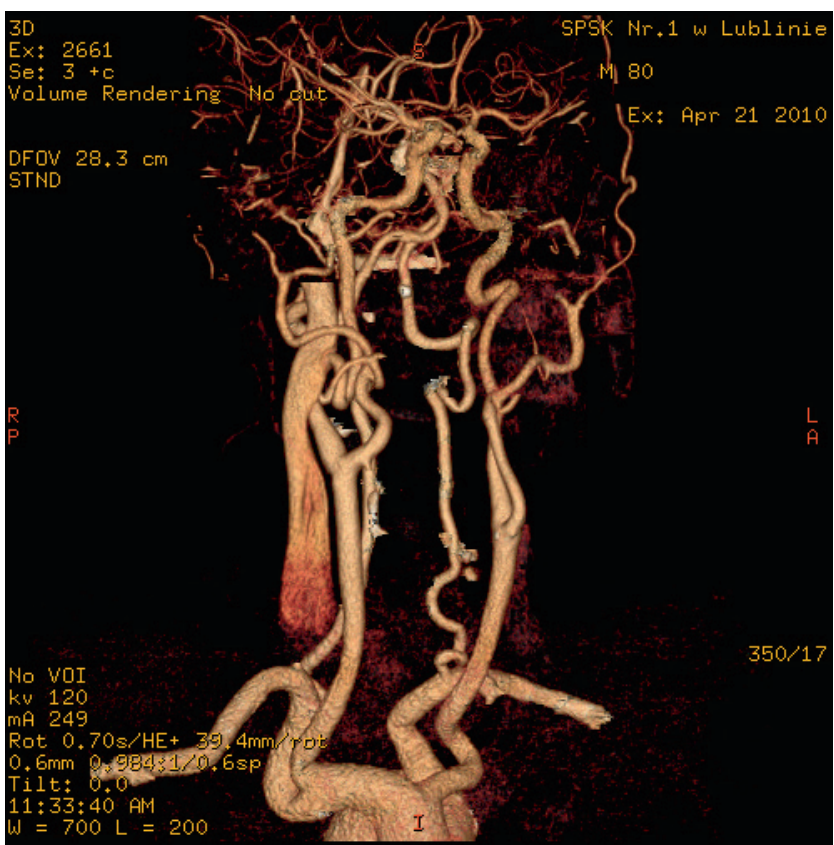

Figure 1. Anterior 3D reconstructed image shows the anomaly of the issue of the left common carotid artery and tortuosity of brain feeding arteries. Note also the double kinkings of the both subclavicular arteries and the extent of the bending of the proximal part of the right common carotid artery

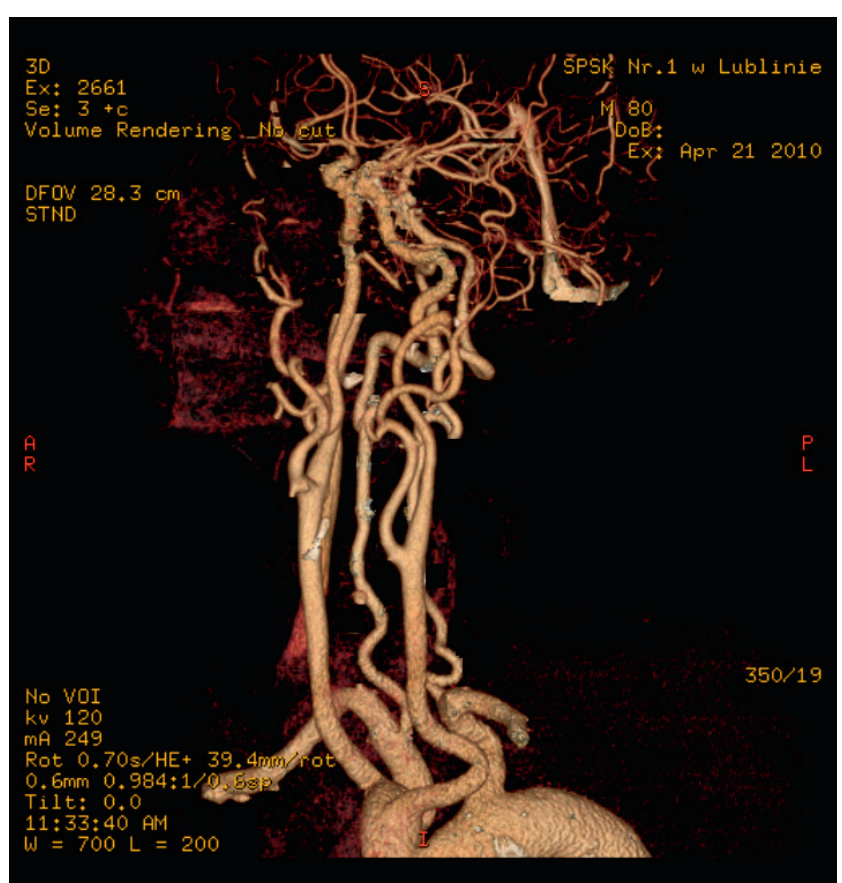

Figure 2. Lateral 3D reconstructed image shows the common brachiocepahlic trunk and the abnormal issue and course of the left common carotid artery with its double kinking. Double and triple kinkings of the vertebral arteries can also be appreciated. The right common carotid artery presents a curved part at the beginning

without significant changes, but then had a threefold kinking (Fig. 3).

The other cervical and intracranial arteries (both internal carotid arteries, the vertebral arteries and the basilar artery) were without significant changes. There were small calcifications in the wall in the intracranial parts of the internal carotid arteries and extracranial parts of vertebral arteries. The Circle of Willis (got) enhanced correctly.

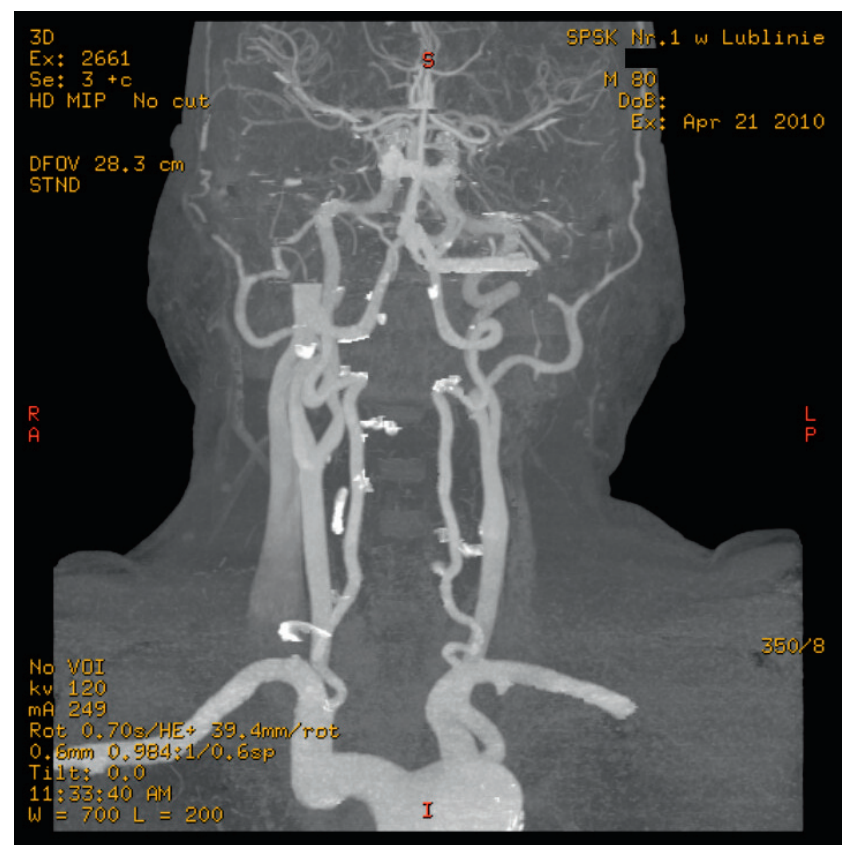

Figure 3. Coronal MIP image shows well triple kinking of the left vertebral artery and double kinking of the right vertebral artery, as well as the abnormalities in carotid arteries. Note the right jugular vein being contrasted before the left one, which indicates slower hemodynamics in brain feeding arteries on the left side, therefore explaining greater hypoperfusion values in the brain on the left side.

Brain CT images showed small, cortical, peripheral, old ischemic changes in both the left and right parietal lobes. A slight cortical and sub-cortical cerebral atrophy was also noted.

Perfusion parameters showed mostly decreased values of blood flow and volume in the left frontal lobe, with generalised decrease on blood parameters in both frontal lobes. This image may be due to the numerous kinking of the carotid and vertebral arteries, especially on the left side, that could have led to the local drop in brain perfusion and therefore the cause of the recurrent fainting.

The patient was discharged from hospital in good health, and further referred to a vascular surgeon who scheduled the patient for future anteposition of the carotid artery.

\section{DISCUSSION}

The clinical entity of elongation and tortuosity of the aorta and the major arteries, secondary to degenerative changes of the blood vessels, usually coexisting with stenosis, is called kinking [8]. Kinking is distinguished from other elongations of arteries - tortuosity and coiling. Tortuosity is an elongation with curving; coiling is an elongation and redundancy of the artery resulting in an exaggerated $\mathrm{S}$-shaped curvature or in a circular configuration. Stenotic kinking is an elongation causing angulation of less than $90^{\circ}$ with Duplex ultrasonography consistent with the artery stenosis of $70 \%$ or greater [9].

Tortuosity of the internal carotid artery is observed in $35 \%$, coiling in $6 \%$, and kinking in $5 \%$ of patients, according to the literature, with a fourfold higher incidence in women $[2,4]$. Kinking can sometimes be the reason for cerebral ischemia, resulting in Transient Ischemic Attack (TIA) and/or completed stroke, usually a minor one. Usually, however, clinical symptoms hardly ever arise, and only some 
of the cases are diagnosed $[8,10]$. Symptomatic patients may complain of having had multiple transitory strokes, probably induced by changes in the position of the head, or extreme positions of the head. Cerebral insufficiency should be evaluated at the physical examination by palpation of the carotid arteries with the patient's head in all possible positions, and the patient checked regularly for blood pressure, state of consciousness, and neurologic deficit $[4,6]$.

Further diagnostic imaging techniques are recommended in all patients who show any indications that kinking might be the cause of stroke. Those include: echo-colour Doppler ultrasound (ECDUS), magnetic resonance imaging (MRI), multidetector-row computed tomography angiography (MDCTA), digital subtraction angiography (DSA), and nuclear medicine imaging [4]. These examinations can even show in what mechanism kinking and coiling can determine neurological symptoms: in a thromboembolic or a haemodynamic mechanism [11].

Results from recent retrospective clinical trials with the use of MDCTA demonstrate that kinking may be associated with symptoms, whereas coiling does not appear to be associated with symptoms. The degree of severity of the carotid artery stenosis is not related to the presence of the vessel tortuosity [4].

Kinking of the carotid artery needs surgical reconstruction when it causes impairments in haemodynamics, with clinical symptoms and neurological signs - transitory ischaemic attack (TIA)/stroke [8].

A recent randomized trial, including patients with symptomatic carotid coiling and kinking, demonstrated considerably better results in periods of relieving neurologic symptoms and preventing carotid thrombosis and stroke after surgery treatment than after only antiplatelet therapy [12]. In such cases, operative treatment of the kinking must be considered [8].

There are many surgical treatments for kinking - from simple dissection and kinking fixation to extensive arterial reconstructions of the carotid bifurcation region [10]. One such treatment is arteriotomy - opening of the lumen and shortening of the carotid artery. Although excellent results are reported in the literature, arteriotomy carries several complications, for example, narrowing of the lumen, impotency of the suture, and postoperative occlusion caused by mural thrombosis [8]. Another one is anteposition of the carotid artery (simple kinking straightening and fixation), which repairs the kinking without arteriotomy; the muscle carefully holds the artery in its new location. In lesser kinkings, a simple surgical technique without cross-clamping is effective, easy, low-risk, and the recovery time is short. The long-term results of surgical treatment are very satisfactory $[8,9,10]$.

At the present, there is no proof to support the extension of this surgical indication to asymptomatic patients with carotid kinking [9].

\section{CONCLUSION}

The presented case shows that an abnormal origin of the left common carotid artery and multiple kinkings may be associated with symptoms of cerebrovascular insufficiency. Clinical symptoms of carotid kinking seldom arise, and only some of the cases are diagnosed. Usually, cerebral insufficiency secondary to kinking of the carotid artery is evaluated at the physical examination, but imaging techniques are valid diagnostic tools for the assessment of carotid anomalies.

It is generally accepted that the kinking of the carotid artery needs surgical reconstruction when it causes impairments in haemodynamics, with clinical symptoms and neurological signs. In asymptomatic patients, there is no indication for surgical treatment. These patients should be checked regularly for blood pressure and neurologic deficit, because carotid anomalies can have important clinical results.

\section{REFERENCES}

1.Paulsen F, Tillmann B, Christofides C, Richter W, Koebke J. Curving and looping of the internal carotid artery in relation to the pharynx: frequency, embryology and clinical implications. J Anat. 2000; 197: 373-381.

2. Das SK, Browand TD, Byrom R. Aortic root anomalies of the neck presenting in adults. Review of the literature with three case reports. Eur J Vasc Endovasc Surg. 2005; 30: 48-51.

3. Poultsides GA, Lolis ED, Vasquez J, MD, Drezner AD, MD, Venieratos D. Common origins of carotid and subclavian arterial systems: report of a rare aortic arch variant. Ann Vasc Surg. 2004; 18(5): 597-600.

4. Saba L, Mallarini G. Correlation between kinking and coiling of the carotid arteries as assessed using MDCTA with symptoms and degree of stenosis. Clin Radiol. 2010; 65: 729-734.

5. Lin PH, Bush RL, Reddy P, Lumsden AB. An unusual cause of dysphagia. Coil of the proximal common carotid artery: a case report. Vasc Surg. 2000; 34: 521-526.

6. Milic DJ, Jovanovic MM, Zivic SS, Jankovic RJ. Coiling of the left common carotid artery as a cause of transient ischemic attacks. J Vasc Surg. 2007; 45: 411-413.

7. Gupta A, Winslet MC. Tortuous common carotid artery as a cause of dysphagia. J R Soc Med. 2005; 98: 275-276.

8. Szekely G, Csecsei GI. Anteposition of the internal carotid artery for surgical treatment of kinking. Surg Neurol. 2001; 56: 124-126.

9. Illuminati G, Ricco J-B, Caliò FG, D'Urso A, Ceccanei G, Vietri F. Results in a consecutive series of 83 surgical corrections of symptomatic stenotic kinking of the internal carotid artery. Surgery. 2008; 143: 134-139.

10. Benes V, Mohapl M. Alternative surgery for the kinked internal carotid artery. Acta Neurochir. 2001; 143: 1267-1272 (Wien).

11. Lee SW, Antiga L, Spence JD, et al. Geometry of the carotid bifurcation predicts its exposure to disturbed flow. Stroke. 2008; 39: 2341-2347.

12. Ballotta E, Thiene G, Baracchini C, et al. Surgical vs medical treatment for isolated internal carotid artery elongation with coiling and kinking in symptomatic patients: a prospective randomized clinical study. J Vasc Surg. 2005; 42: 838-846. 